\title{
Natural Convection Inside Vertical Isothermal Ducts of Constant Arbitrary Cross Section
}

\author{
M. M. Yovanovich* and P. Teertstra ${ }^{\dagger}$ \\ University of Waterloo, Waterloo, Ontario N2L 3G1, Canada \\ and \\ Y. S. Muzychka \\ Memorial University of Newfoundland, St. John's, NewfoundlandA1B 3X5, Canada
}

\begin{abstract}
A model for steady laminar natural convection inside vertical, arbitrarily shaped isothermal ducts of constant cross section is presented. The model is based on a combination of asymptotic solutions for fully developed and developing, boundary-layer flow. The fully developed asymptote model utilizes forced convection, internal flow modeling techniques, and an approximate model is presented, which is valid for a wide range of duct shapes and aspect ratios. The model is validated using experimental data and numerical results from the literature for a variety of duct shapes, including the triangle, square, circle, rectangle and the special case of parallel plates. The model is in good agreement with all of the data, with an rms percent difference of $10 \%$ or less in most cases.
\end{abstract}

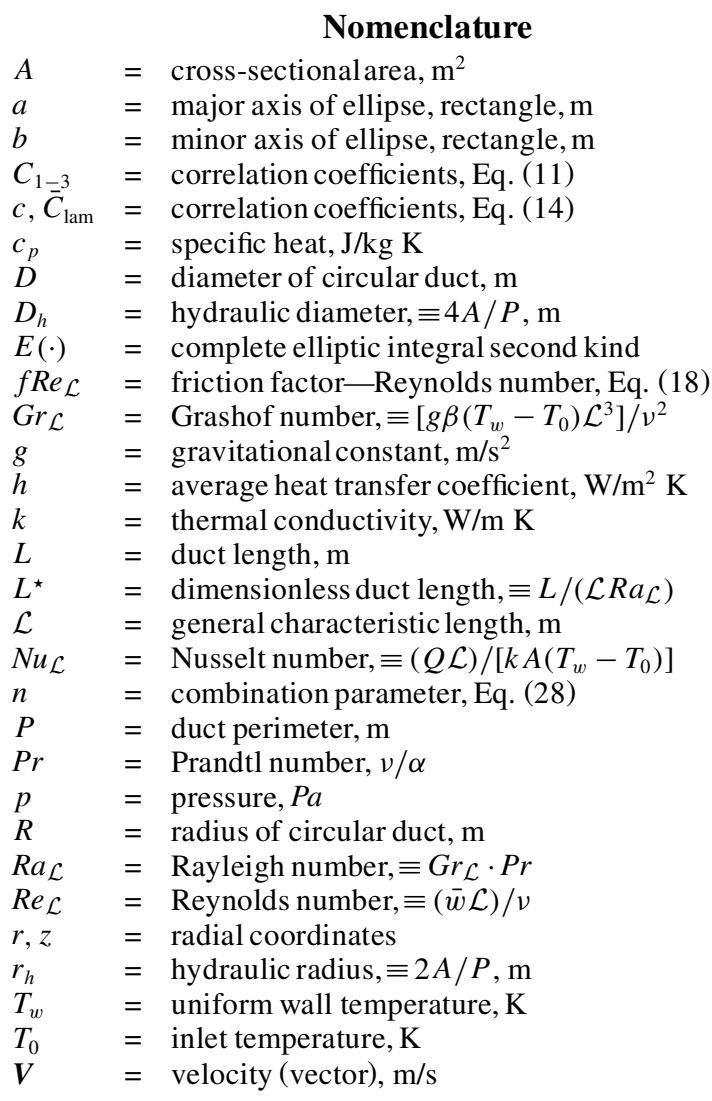

Presented as Paper 2001-0368 at the AIAA 39th Aerospace Sciences Meeting, Reno, NV, 8-11 January 2001; received 23 March 2001; revision received 29 August 2001; accepted for publication 29 August 2001. Copyright (C) 2001 by the authors. Published by the American Institute of Aeronautics and Astronautics, Inc., with permission. Copies of this paper may be made for personal or internal use, on condition that the copier pay the $\$ 10.00$ per-copy fee to the Copyright Clearance Center, Inc., 222 Rosewood Drive, Danvers, MA 01923; include the code 0887-8722/02 \$10.00 in correspondence with the CCC.

* Distinguished Professor Emeritus, Microelectronics Heat Transfer Laboratory, Department of Mechanical Engineering. Fellow AIAA.

${ }^{\dagger}$ Graduate Research Assistant, Microelectronics Heat Transfer Laboratory, Department of Mechanical Engineering.

${ }^{*}$ Assistant Professor, Faculty of Engineering and Applied Science.

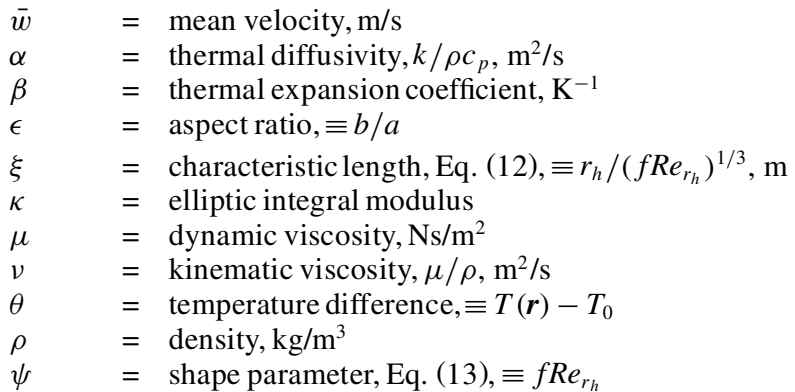

Subscripts

$\sqrt{ } A=$ based on characteristiclength $\sqrt{ } A$

bl = boundary layer

$D_{h} \quad=$ based on characteristiclength $D_{h}$

fd $\quad=$ fully developed

$r_{h} \quad=$ based on characteristiclength $r_{h}$

$w \quad=$ wall

$0 \quad=$ inlet

Superscripts

$C \quad=$ circular

$P \quad=$ polygonal

\section{Introduction}

S TEADY natural convection inside isothermal vertical ducts of constant cross section of arbitrary shape is a fundamental problem that occurs in a variety of applications. The problem of interest, as shown in Fig. 1, consists of a vertical duct of length $L$, constant perimeter $P$, and uniform cross-sectionalarea $A$. The inner wall of the duct is maintained at a uniform temperature $T_{w}$, and the fluid temperature at the duct inlet located at $z=0$ is $T_{0}$. Assuming that $T_{w}>T_{0}$, there will be a buoyancy-driven flow through the duct in the positive $z$ direction, and convection heat transfer will occur from the duct wall into the fluid.

A number of researchers have published experimental and numerical data in the literature for various cross-sectional shapes. Elenbaas ${ }^{1}$ presented experimental measurements for a wide variety of cross sections, including the triangular, circular, and square ducts, as well as the 2:1 and 5:1 rectangular ducts. For the circular duct Davis and Perona ${ }^{2}$ report the results of numerical simulations, whereas Dyer ${ }^{3}$ presents experimentaldata. In the case of the square duct, Ramakrishna et al. ${ }^{4}$ present data obtained from a numerical 


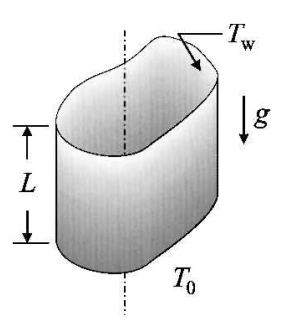

Fig. 1 Schematic of physical problem.
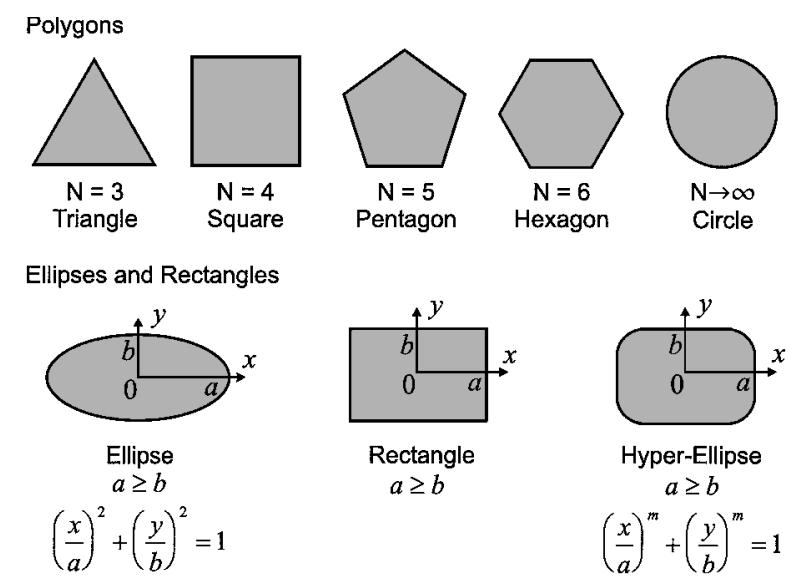

Fig. 2 Duct cross sections.

study. The remaining studies are limited to the vertical parallel plates, such as the experimentalmeasurements of Elenbaas ${ }^{5}$ and the numerical results of Miyatake and Fujii ${ }^{6}$ and Ofi and Hetherington. ${ }^{7}$

There are three models currently available in the literature for natural convection in vertical isothermal ducts: Elenbaas, ${ }^{1}$ Aihara, ${ }^{8}$ and Raithby and Hollands. ${ }^{9}$ Each of these models is applicable for noncircular ducts through the use of the hydraulic radius as the characteristic length:

$$
r_{h}=2 A / P
$$

However, these models are applicable only for ducts with crosssectional aspect ratio approximately equal to one; for the small aspect ratio rectangular or elliptical ducts the predictions of these models can vary significantly from the available empirical results. There are currently no models available for arbitrarily shaped ducts for the full range of aspect ratio from unity, that is, circular duct to zero or parallel plates.

The objective of the current study is to develop a natural convection model for the full range of the Rayleigh number for arbitrarily shaped, singly connected constant cross-section ducts over the full range of aspect ratio, $0<\epsilon \leq 1$, as shown in Fig. 2. The model will be validated using the available experimental and numerical data for a variety of duct shapes, including the limiting case of the vertical parallel plates.

\section{Problem Description}

The governingequationsfor the problem of interest—natural convection in a vertical, isothermal duct with uniform cross sectioncan be expressed in vector notation as follows:

Continuity:

$$
\nabla \cdot \boldsymbol{V}=0
$$

Momentum:

$$
\rho \boldsymbol{V} \cdot \nabla \boldsymbol{V}=-g \beta \theta+\mu \nabla^{2} \boldsymbol{V}
$$

Energy:

$$
\boldsymbol{V} \cdot \nabla \theta=\alpha \nabla^{2} \theta
$$

with temperature difference $\theta=T(\boldsymbol{r})-T_{0}$. The boundary conditions at the inlet are

$$
\boldsymbol{V}=0, \quad \theta=0
$$

and at the duct walls

$$
\boldsymbol{V}_{w}=0, \quad \theta=\theta_{w}=T_{w}-T_{0}
$$

The total heat-transfer rate from the duct walls to the fluid is

$$
Q=\iint_{A}-\left.k \frac{\partial \theta}{\partial n}\right|_{w} \mathrm{~d} A
$$

where the heat-transfer area is $A=P L$. The dimensionless heattransfer rate is characterized by the Nusselt number:

$$
N u_{\mathcal{L}}=\frac{Q \mathcal{L}}{k A\left(T_{w}-T_{0}\right)}
$$

The independent variables are nondimensionalized using the Rayleigh number, defined using the general scale length:

$$
R a_{\mathcal{L}}=\frac{g \beta\left(T_{w}-T_{0}\right) \mathcal{L}^{3}}{\alpha \nu}
$$

For the natural convection duct problem it is convenient to modify the Rayleigh number by the aspect ratio, expressed as a function of the general scale length parameter $\mathcal{L}$. The resulting dimensionless parameter, sometimes called the channel Rayleigh number, is

$$
R a_{\mathcal{L}} \frac{\mathcal{L}}{L}=\frac{g \beta\left(T_{w}-T_{0}\right) \mathcal{L}^{3}}{\alpha \mathcal{V}} \frac{\mathcal{L}}{L}
$$

The dimensionless duct length is defined as the inverse of this modified Rayleigh number:

$$
L^{\star}=L / \mathcal{L} R a_{\mathcal{L}}
$$

\section{Review of Previous Models}

Elenbaas ${ }^{1}$ is widely regarded as the first to study natural convection in ducts. He developed a model, validated by experimental data for a wide range of duct cross sections, based on the asymptotic solutions of fully developed and developing flow. Assuming that, for fully developed flow $\theta(r) \rightarrow \theta_{w}$ as $L^{\star} \rightarrow \infty$, the following relation for the area average heat-transfer coefficient for the circular duct was obtained:

$$
\frac{h R}{k}=\frac{\rho c_{p} \bar{w}}{k} \frac{A R}{P L}, \quad \bar{w}=\frac{g \beta \theta_{w} R^{2}}{8 v}
$$

For fully developed flow in the circular duct of radius $R$, Elenbaas ${ }^{1}$ reported the following Nusselt-Rayleigh number relation:

$$
N u_{R}=h R / k=\frac{1}{16}(R / L) R a_{R}
$$

For noncircular ducts the hydraulic radius was recommended as the scale length $\mathcal{L}=r_{h}=2 A / P$, and the relation recommendedfor fully developed flow for ducts of noncircular cross section was

$$
N u_{r_{h}}=\left(1 / f R e_{r_{h}}\right)\left(r_{h} / L\right) R a_{r_{h}}
$$

where the friction factor Reynolds number product $f R e_{r_{h}}$ can be obtained from forced laminar flow studies.

The solution for natural convection from an isothermal vertical flat was used for the developing flow asymptote $L^{\star} \rightarrow 0$ :

$$
N u_{L}=0.60 R a_{L}^{\frac{1}{4}}
$$

where the scale length for this asymptote is the plate height $\mathcal{L}=L$. For intermediate values of the flow parameter $0<L^{\star}<\infty$, Elenbaas ${ }^{1}$ developed the following combined relationship for arbitrarily shaped ducts:

$$
N u_{r_{h}}=\frac{G r_{r_{h}} \operatorname{Pr}}{f \operatorname{Re}_{r_{h}}} \frac{r_{h}}{L}\left[1-\exp \left\{-\left(\frac{C_{1}}{G r_{r_{h}} \operatorname{Pr}} \frac{L}{r_{h}}\right)^{C_{2}}\right\}\right]^{C_{3}}
$$


Because Elenbaas used two different scale lengths, it was necessary for him to find a means of combining the asymptotic results into a comprehensive model. This was accomplished by a judicious choice of the coefficients $C_{2}$ and $C_{3}$, which must satisfy the condition $C_{2} C_{3}=\frac{3}{4}$.

Elenbaas obtained experimental air data for ducts with a variety of cross sections, including triangular, circular, square, rectangular with aspect ratios 2:1 and 5:1, and parallelplates. Elenbaas observed that the use of the hydraulic radius as the scale length produced a family of parallel curves for the different cross sections for small values of the independent parameter $\left(r_{h} / L\right) R a_{r_{h}}<10$ and that all curves converged for large values $\left(r_{h} / L\right) R a_{r_{h}}>500$.

Aihara ${ }^{8}$ reviewed the work of Elenbaas ${ }^{1}$ and developed a model in which both the Nusselt and Rayleigh numbers are based on a new scale length:

$$
\xi=r_{h} /\left(f \operatorname{Re}_{r_{h}}\right)^{\frac{1}{3}}
$$

Using this scale length, Aihara ${ }^{8}$ modified the correlation equation of Elenbaas ${ }^{1}$ as follows:

$$
N u_{r_{h}}=\frac{G r_{r_{h}} \operatorname{Pr}}{\psi} \frac{r_{h}}{L}\left[1-\exp \left\{-\psi\left(\frac{0.5}{G r_{r_{h}} \operatorname{Pr}} \frac{L}{r_{h}}\right)^{\frac{3}{4}}\right\}\right]
$$

where the shape parameter was defined as the friction factorReynolds number product, $\psi=f R e_{r_{h}}$. By recasting the experimental data of Elenbaas ${ }^{1}$ in terms of $N u_{\xi}$ and $(\xi / L) R a_{\xi}$, Aihara ${ }^{8}$ demonstrated that the data for very small and very large values of $(\xi / L) R a_{\xi}$ approached a single curve for all duct cross sections including the parallel plates data.

Raithby and Hollands ${ }^{9}$ proposed the following comprehensive model for arbitrary cross sections based on the Churchill and Usagi method $^{10}$ of combining asymptotic solutions:

$$
N u_{r_{h}}=\left\{\left(\frac{R a_{r_{h}}}{f R e_{r_{h}}}\right)^{-1.5}+\left[c \bar{C}_{\mathrm{lam}}\left(\frac{r_{h}}{L} R a_{r_{h}}\right)^{\frac{1}{4}}\right]^{-1.5}\right\}^{-\frac{1}{1.5}}
$$

valid over the range $\left(r_{h} / L\right) R a_{r_{h}} \leq 10^{4}$, where $\bar{C}_{\text {lam }}=0.62$ for air $(\operatorname{Pr}=0.71)$. Through a fit of the available empirical data, the value for the correlation coefficient $c$ was determined: $c=1.17$.

The correlation equations of Raithby-Hollands ${ }^{9}$ and Elenbaas ${ }^{1}$ for the circular duct with air cooling are in relatively close agreement, with differences in the range of $2-9 \%$, for small and large values of the duct Rayleigh number, while a relatively large difference of up to $25 \%$ appears in the transition region.

\section{Model Development}

\section{Asymptotic Limits}

This problem of fully developed flow in constant cross sections has been analyzed in the forced convection literature quite extensively, such as Shah and London. ${ }^{11}$ Assuming negligible inertial forces, the momentum equation for an arbitrarily shaped cross section reduces to

$$
\nabla^{2} w=-g \beta \theta_{w} / v
$$

Thus, the mean natural convection velocity can be obtained by solving the momentum equation (15) only. Equating Eq. (15) with the classical momentum equation for fully developed forced duct flow

$$
\nabla^{2} w=\frac{1}{\mu} \frac{\mathrm{d} p}{\mathrm{~d} z}
$$

gives the following relationship for the axial pressure gradient:

$$
\frac{\mathrm{d} p}{\mathrm{~d} z}=-\rho g \beta \theta_{w}
$$

Combining the friction factor-Reynolds number product $f R e$, defined as

$$
f \operatorname{Re}_{\mathcal{L}}=2 \frac{(A / P)(-\mathrm{d} p / \mathrm{d} z) \mathcal{L}}{\mu \bar{w}}
$$

with Eq. (17) results in the following expressionfor the mean natural convection velocity for fully developed flow $\bar{w}$ :

$$
\bar{w}=2 \frac{(A / P) \rho g \beta \theta_{w} \mathcal{L}}{\mu f R e_{\mathcal{L}}}
$$

Assuming that, for fully developed flow $\theta(r) \rightarrow \theta_{w}$ as $L^{\star} \rightarrow \infty$, an energy balance over the full length $L$ of the duct gives

$$
h(P L) \theta_{w}=\rho \bar{w} A c_{p} \theta_{w}
$$

Nondimensionalizingusing the Nusseltnumberbased on the general scale length $\mathcal{L}$ and combining with the mean velocity, Eq. (19), gives the following expression for the fully developed flow asymptote:

$$
N u_{\mathcal{L}}=\frac{h \mathcal{L}}{k}=2 \frac{R a_{\mathcal{L}}(\mathcal{L} / L)}{f R e_{\mathcal{L}}}\left(\frac{A}{P \mathcal{L}}\right)^{2}
$$

Using the hydraulic diameter $D_{h}=4 A / P$ as the scale length, Eq. (21) reduces to

$$
N u_{f d}=\frac{R a_{D_{h}}\left(D_{h} / L\right)}{8 f R e_{D_{h}}}
$$

When the square root of the cross-sectionalarea is used as the scale length $\mathcal{L}=\sqrt{ } A$, Eq. (21) becomes

$$
N u_{f d}=2 \frac{R a_{\sqrt{A}}(\sqrt{A} / L)}{f R e_{\sqrt{A}}}\left(\frac{\sqrt{A}}{P}\right)^{2}
$$

Muzychka ${ }^{12}$ demonstrated that the use of $\sqrt{ } A$ as the scale length is preferable because the group $f R e_{\sqrt{ } A}$ is a very weak function of shape and only depends upon the aspect ratio of the duct. Table 1 shows values of the parameter $f R e$ for both $D_{h}$ and $\sqrt{ } A$ length scales for the polygonal duct shapes. The variation between the triangular duct and the circular duct has been reduced to approximately $7 \%$ from $17 \%$ when $\sqrt{ } A$ is chosen as the characteristic length. This behavior is also evident for elongated ducts, such as the rectangular and elliptic cross sections. Table 2 shows the results for both length

\begin{tabular}{|c|c|c|c|c|}
\hline$N$ & $f R e_{D_{h}}^{11}$ & $\left(\frac{f R e^{P}}{f R e^{C}}\right)_{D_{h}}$ & $f R e_{\sqrt{ } A}^{12}$ & $\left(\frac{f R e^{P}}{f R e^{C}}\right)$ \\
\hline 3 & 13.33 & 0.833 & 15.19 & 1.071 \\
\hline 4 & 14.23 & 0.889 & 14.23 & 1.004 \\
\hline 5 & 14.73 & 0.921 & 14.04 & 0.990 \\
\hline 6 & 15.05 & 0.941 & 14.01 & 0.988 \\
\hline 7 & 15.31 & 0.957 & 14.05 & 0.991 \\
\hline 8 & 15.41 & 0.963 & 14.03 & 0.989 \\
\hline 9 & 15.52 & 0.970 & 14.04 & 0.990 \\
\hline 10 & 15.60 & 0.975 & 14.06 & 0.992 \\
\hline 20 & 15.88 & 0.993 & 14.13 & 0.996 \\
\hline$\infty$ & 16 & 1.000 & 14.18 & 1.000 \\
\hline
\end{tabular}
scales for the rectangular and elliptic ducts. Again, the variation is reduced to approximately $7 \%$ from $31 \%$.

Muzychka ${ }^{12}$ also showed that many other complex duct shapes are easily predicted within $\pm 10 \%$ using Eq. (24), as shown in Fig. 3 for a variety of noncircular ducts. Also included in Fig. 3 is the exact closed-form solution for the elliptic cross section, which can be used as an approximation for other duct shapes. The $f R e_{\sqrt{ } A}$ for the elliptic duct is given by ${ }^{12}$

$$
f \operatorname{Re}_{\sqrt{A}}=8 \sqrt{\pi}\left[\frac{\pi}{4} \frac{\left(1+\epsilon^{2}\right)}{\sqrt{\epsilon} \boldsymbol{E}\left(\sqrt{1-\epsilon^{2}}\right)}\right]
$$

Table $1 f R e$ results for polygonal geometries 
Table $2 f R e$ results for elliptical and rectangular ducts

\begin{tabular}{|c|c|c|c|c|c|c|}
\hline \multirow[b]{2}{*}{$b / a$} & \multicolumn{3}{|c|}{$f R e_{D_{h}}^{11}$} & \multicolumn{3}{|c|}{$f \operatorname{Re}_{\sqrt{ } A}^{12}$} \\
\hline & Rectangular & Elliptical & $\left(\frac{f R e^{R}}{f R e^{E}}\right)_{D_{h}}$ & Rectangular & Elliptical & $\left.\frac{f R e^{R}}{f R e^{E}}\right)$ \\
\hline 0.01 & 23.67 & 19.73 & 1.200 & 119.56 & 111.35 & 1.074 \\
\hline 0.05 & 22.48 & 19.60 & 1.147 & 52.77 & 49.69 & 1.062 \\
\hline 0.10 & 21.17 & 19.31 & 1.096 & 36.82 & 35.01 & 1.052 \\
\hline 0.20 & 19.07 & 18.60 & 1.025 & 25.59 & 24.65 & 1.038 \\
\hline 0.30 & 17.51 & 17.90 & 0.978 & 20.78 & 20.21 & 1.028 \\
\hline 0.40 & 16.37 & 17.29 & 0.947 & 18.12 & 17.75 & 1.021 \\
\hline 0.50 & 15.55 & 16.82 & 0.924 & 16.49 & 16.26 & 1.014 \\
\hline 0.60 & 14.98 & 16.48 & 0.909 & 15.47 & 15.32 & 1.010 \\
\hline 0.70 & 14.61 & 16.24 & 0.900 & 14.84 & 14.74 & 1.007 \\
\hline 0.80 & 14.38 & 16.10 & 0.893 & 14.47 & 14.40 & 1.005 \\
\hline 0.90 & 14.26 & 16.02 & 0.890 & 14.28 & 14.23 & 1.004 \\
\hline 1.00 & 14.23 & 16.00 & 0.889 & 14.23 & 14.18 & 1.004 \\
\hline
\end{tabular}

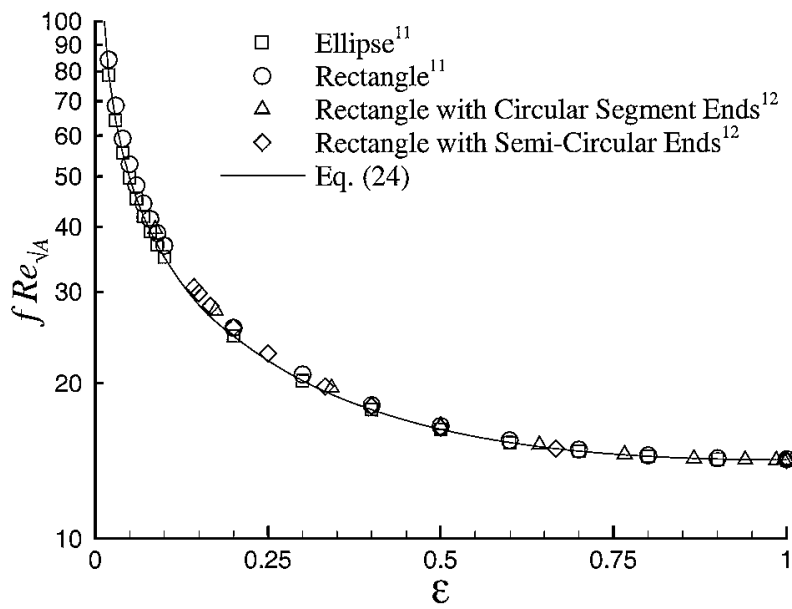

Fig. $3 f R e_{\sqrt{A}}$ for various duct cross sections.

where $\boldsymbol{E}(\cdot)$ is the complete elliptic integral of the second kind and $0.01<\epsilon=b / a \leq 1$ is the aspectratio of the duct. Although $E(\kappa)$ can be computed by polynomial approximations and series expansions for small and large arguments, ${ }^{13}$ the following expression provides accurate approximations to within $0.2 \%$ for all $\epsilon>0.001$ :

$$
E\left(\sqrt{1-\epsilon^{2}}\right)=\frac{\pi}{4}(1+\epsilon) \frac{\left\{64-3\left[(1-\epsilon)^{4} /(1+\epsilon)^{4}\right]\right\}}{\left\{64-16\left[(1-\epsilon)^{2} /(1+\epsilon)^{2}\right]\right\}}
$$

As shown in Tables 1 and 2, the maximum difference between the exact values of $f R e_{\sqrt{ } A}$ presented in Shah and London ${ }^{11}$ for common duct shapes and the model is reduced from approximately 17 to $7 \%$.

The boundary-layerlimit based on the duct length as scale length given by Elenbaas ${ }^{1}$ is

$$
N u_{L}=0.6 R a_{L}^{\frac{1}{4}}
$$

Substituting $\sqrt{ } A$ as the scale length gives

$$
N u_{\mathrm{b} 1}=0.6\left[R a_{\sqrt{A}}(\sqrt{A} / L)\right]^{\frac{1}{4}}
$$

\section{Composite Model}

A composite model using the method of Churchill and Usagi ${ }^{10}$ is developed by combining the asymptotic solutions for fully developed and boundary-layerflow in the following manner:

$$
N u_{\sqrt{A}}=\left[\left(N u_{\mathrm{fd}}\right)^{-n}+\left(N u_{\mathrm{bl}}\right)^{-n}\right]^{-1 / n}
$$

Substituting the relationships from Eqs. (23) and (27) into the general expression yields

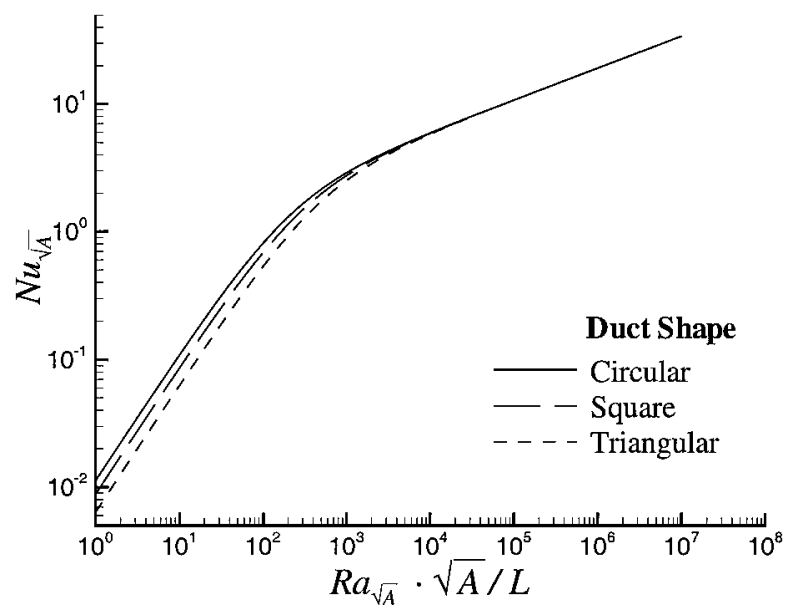

Fig. 4 Model for polygonal ducts.

$$
\begin{aligned}
N u_{\sqrt{A}} & =\left\{\left[2 \frac{R a_{\sqrt{A}}(\sqrt{A} / L)}{f R e_{\sqrt{A}}}\left(\frac{\sqrt{A}}{P}\right)^{2}\right]^{-n}\right. \\
+ & {\left.\left[0.6\left(R a_{\sqrt{A}} \frac{\sqrt{A}}{L}\right)^{\frac{1}{4}}\right]^{-n}\right\}^{-1 / n} }
\end{aligned}
$$

where $n \approx 1.25$ was found to minimize the rms difference of the available data when compared with the proposed model. A combination parameter, which is a function of aspect ratio, can be used to obtain greater accuracy for a wide range of duct shapes and aspect ratio. Raithby and Hollands ${ }^{9}$ recommend values of $n=1.03$ for the circular duct, $n=1.9$ for the parallel plates, and $n=1.5$ for noncircular shapes.

The model predictionsare shown in Fig. 4 for the full range of the independent parameter $R a_{\sqrt{ } A}(\sqrt{ } A / L)$ for the following polygonal shaped ducts: triangle, square, and circular. The model is clearly independent of geometry at the boundary-layerlimit, and the variation between the model curves for fully developed flow for the full range of polygonal duct shapes, $3 \leq N<\infty$, has been minimized by the use of $\sqrt{ } A$ as the scale length.

Figure 5 shows the model predictions for the rectangular ducts having aspect ratios in the range $1 \geq \epsilon \geq 0.01$, corresponding to the square duct and parallel plate channel, respectively. Once again, the model is geometrically independent at the boundary-layerlimit, but the changes in aspect ratio result in large variations between the model curves at the fully developed flow limit.

\section{Model Validation}

The model for the area-average Nusselt number for arbitrarily shaped, constant cross-section isothermal ducts is validated using experimental and numerical data from the literature for a range of 
Table 3 Validation data for ducts

\begin{tabular}{|c|c|c|c|}
\hline Shape & $\sqrt{ } A / r_{h}$ & $f R e_{\sqrt{ } A}$ & $4 \sqrt{ } A / P$ \\
\hline Triangle $^{1}$ & 2.280 & 15.19 & 0.877 \\
\hline Square ${ }^{1,4}$ & 2 & 14.23 & 1 \\
\hline Circle $^{1,2,3}$ & 1.772 & 14.18 & 1.128 \\
\hline 2:1 Rectangle ${ }^{1}$ & 2.121 & 15.55 & 0.943 \\
\hline 5:1 Rectangle ${ }^{1}$ & 2.683 & 19.05 & 0.745 \\
\hline
\end{tabular}

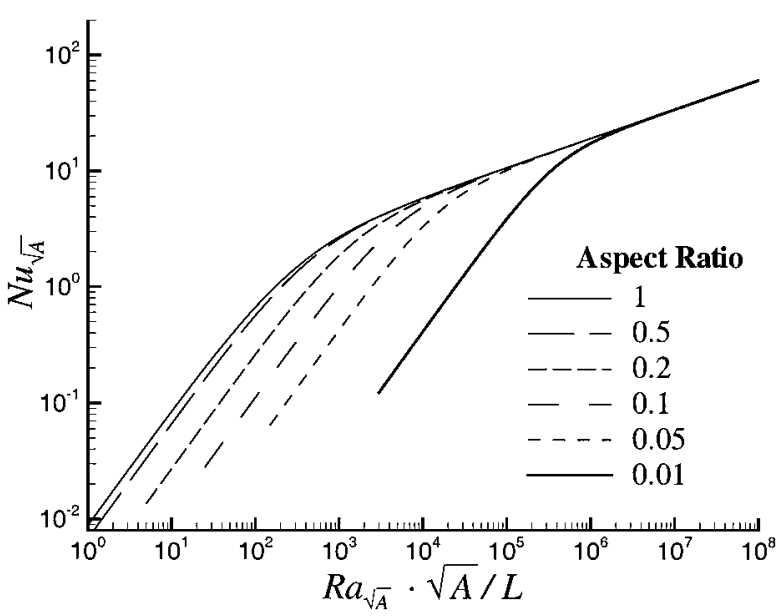

Fig. 5 Model for rectangular ducts.

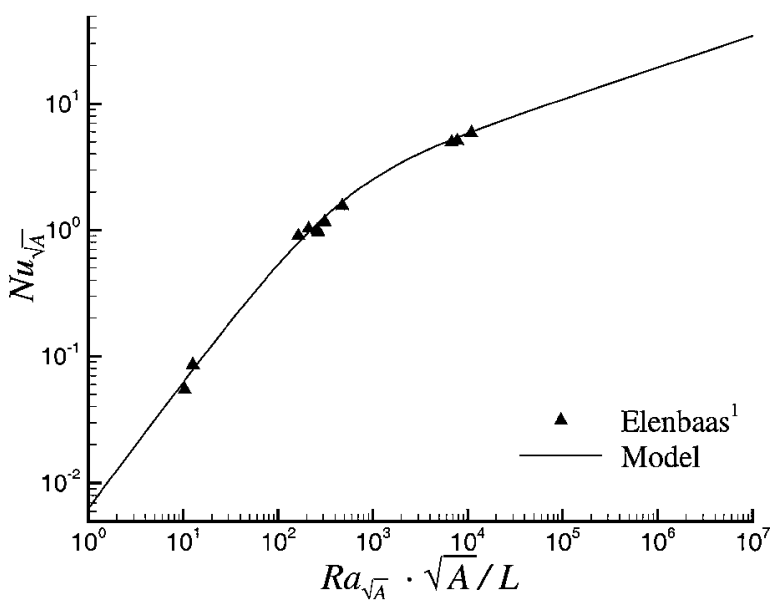

Fig. 6 Model validation for triangular ducts.

different duct shapes, as shown in Table 3 and in the following: the numbers for parallel plates ${ }^{5-7}$ are $10(\sqrt{ } A / W), 119.56\left(f R e_{\sqrt{ } A}\right)$, and $0.198(4 \sqrt{ } A / P)$. To facilitate this comparison, the data are recast in terms of the scale length $\sqrt{ } A$ using the following conversions:

$$
\begin{gathered}
N u_{\sqrt{A}}=N u_{\mathcal{L}}(\sqrt{A} / \mathcal{L}) \\
R a_{\sqrt{A}}(\sqrt{A} / L)=R a_{\mathcal{L}}(\mathcal{L} / L)(\sqrt{A} / \mathcal{L})^{4}
\end{gathered}
$$

In all cases the data presented in the literature were nondimensionalized based on the hydraulic radius $\mathcal{L}=r_{h}$, and the conversion factor $\sqrt{ } A / r_{h}$ is shown in Table 3 . The parallel plate channel is treated as a 100:1 aspect ratio rectangularduct. In the fully developed limit of forced convection, this is a very good approximation, as demonstrated by Muzychka. ${ }^{12}$ The data presented in the literature use the channel width $W$ as the scale length and are recast in terms of $\sqrt{ } A$ using Eqs. (30) and (31) using the conversions factors given previously.

The model, Eq. (29) with $n=1.25$, is compared to the available data for the polygonal ducts - the triangle, square, and circle-in Figs. 6-8. In Fig. 6 the model is shown to be in good agreement with the experimental data of Elenbaas ${ }^{1}$ with an rms difference of $9 \%$. Figure 7 compares the numerical data of Ramakrishna et al. ${ }^{4}$

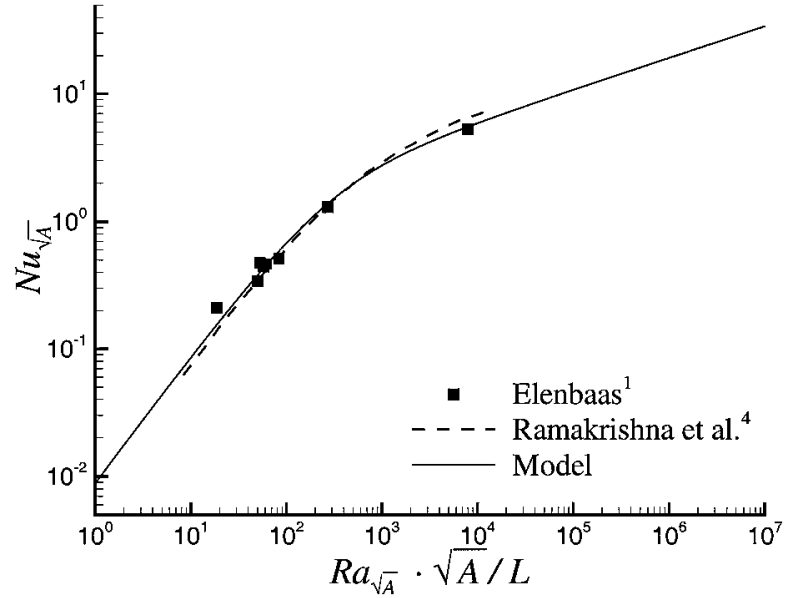

Fig. 7 Model validation for square ducts.

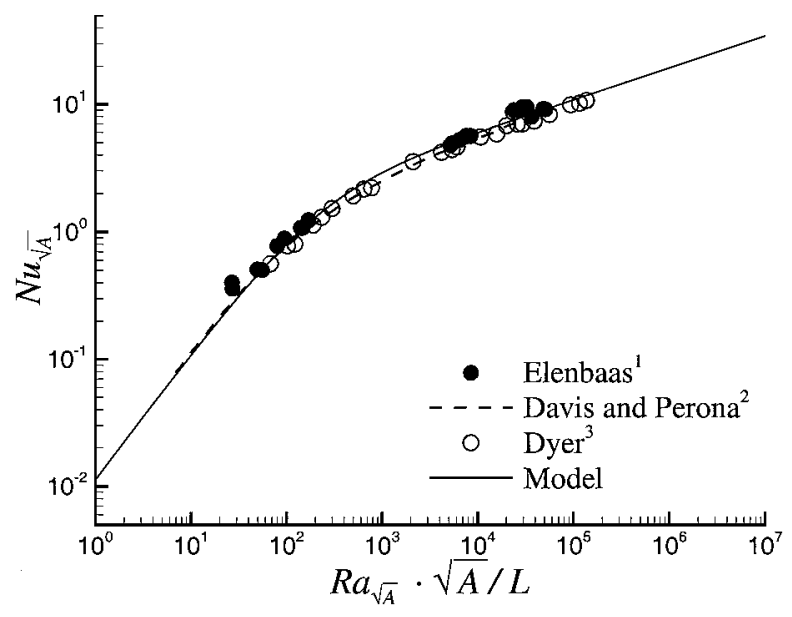

Fig. 8 Model validation for circular ducts.

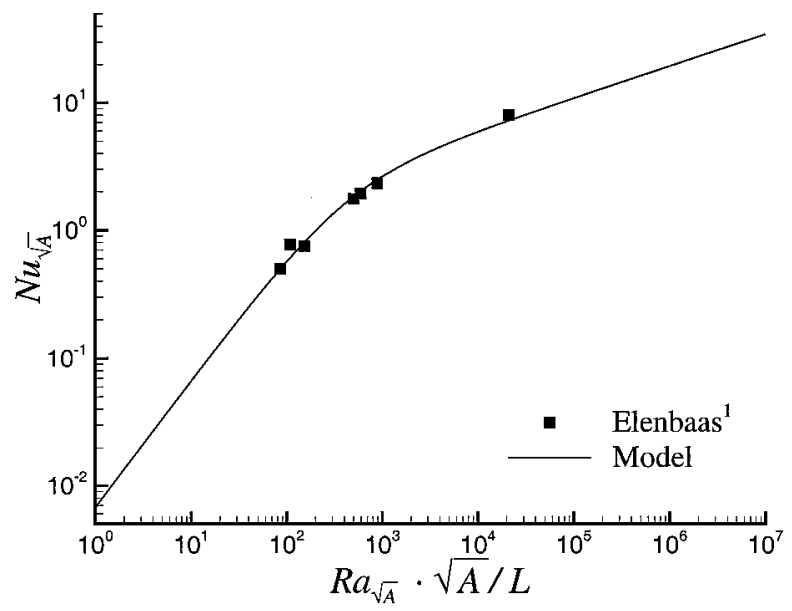

Fig. 9 Model validation for 2:1 rectangular ducts.

and the measurements of Elenbaas ${ }^{1}$ with the model, where the rms percent difference between these two data sets and the model are 10 and $13 \%$, respectively. For the circular duct, as shown in Fig. 8, validation with the numerical data of Davis and Perona ${ }^{2}$ and the experimental data of Dyer ${ }^{3}$ and Elenbaas ${ }^{1}$ shows an average rms difference between the available data and the model of $10 \%$.

For the rectangular ducts a combination coefficient is recommended, which is a function of the aspect ratio of the duct. Based on the combination parameter value $n=2$ commonly used in the literature for the isothermal parallel plate channe ${ }^{14}$ and values of $n$ that minimize the rms percent difference between the model and the data for the square and the 2:1 and 5:1 rectangular ducts, the following correlation is recommended for the combination parameter as a 


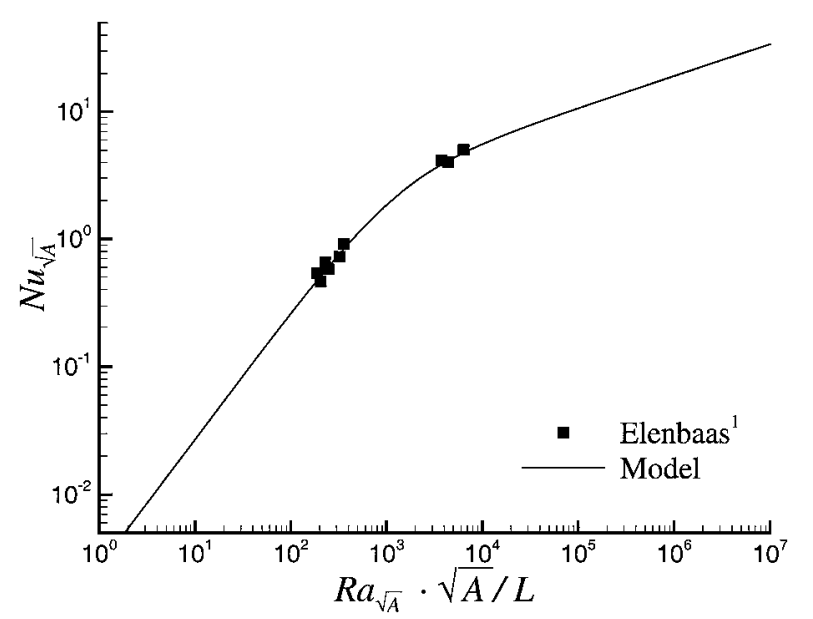

Fig. 10 Model validation for 5:1 rectangular ducts.

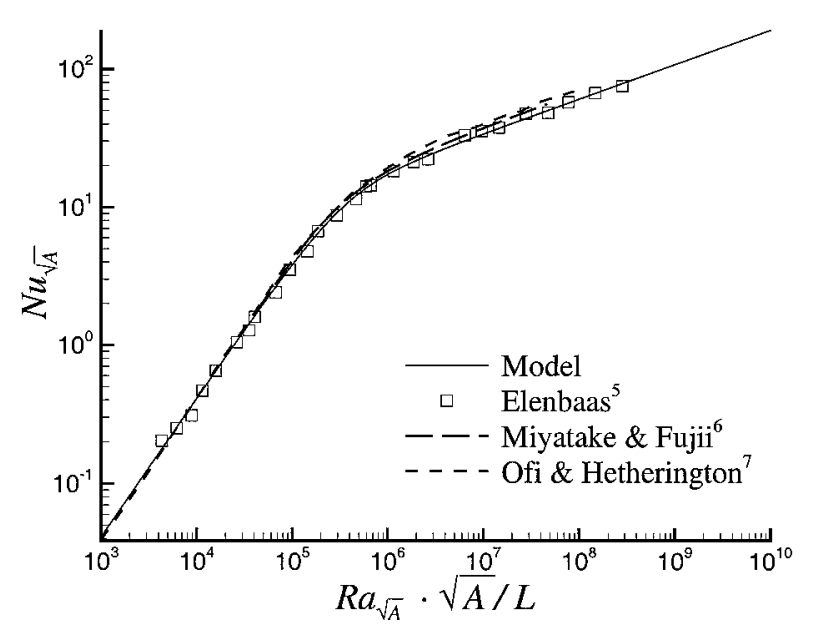

Fig. 11 Model validation for parallel plate channels.

function of the aspect ratio:

$$
n=1.2 / \epsilon^{\frac{1}{9}}
$$

The model is validated with the experimental data of Elenbaas ${ }^{1}$ for rectangular ducts of aspect ratio $2: 1$ and 5:1, as shown in Figs. 9 and 10 , where the rms difference between the data and the model is 9 and $8 \%$, respectively. For the limiting case of the parallel plate channel, the rectangular channel model with $\epsilon=0.01$ is compared with the experimental data of Elenbaas ${ }^{5}$ and the numerical results of Miyatake and Fujii ${ }^{6}$ and Ofi and Hetherington, ${ }^{7}$ as shown in Fig. 11. The rms difference between the measured data of Elenbaas ${ }^{5}$ and the model is $6 \%$, whereas the average rms difference for the numerical results is $9 \%$.

\section{Conclusions}

A model is developed for steady natural convection in isothermal vertical ducts of constant cross section of arbitrary shape. The model combines asymptotic solutions of fully developed flow and developing, boundary-layerflow into a single expression [Eq. (29)], valid for the full range of the modified Rayleigh number. The fully developed flow asymptote is based on forced convectioninternal flow analyses. An approximate model for the friction factor-Reynolds number product for polygonal and rectangular ducts is presented [Eq. (24)], where the elliptic integral can be approximated by Eq. (25). For rectangular or elliptic ducts with aspect ratios less than unity, a correlation of the combination parameter is provided [Eq. (32)]. The model has been validated using the available experimental and numerical data from the literature, with a typical rms difference of $10 \%$ or less.

\section{Acknowledgment}

The authors acknowledge the continued financial support of the Natural Science and Engineering Research Council of Canada under Grant A7445.

\section{References}

${ }^{1}$ Elenbaas, W., "The Dissipation of Heat by Free Convection: The Inner Surface of Vertical Tubes of Different Shapes of Cross-Section," Physica, Vol. 9, No. 8, 1942, pp. 865-874.

${ }^{2}$ Davis, L. P., and Perona, J. J., "Development of Free Convection Flow of a Gas in a Heated Vertical Open Tube," International Journal of Heat and Mass Transfer, Vol. 14, No. 6, 1971, pp. 889-903.

${ }^{3}$ Dyer, J. R., "Natural-Convective Flow Through a Vertical Duct with a Restricted Entry," International Journal of Heat and Mass Transfer, Vol. 21, No. 10, 1978, pp. 1341-1354.

${ }^{4}$ Ramakrishna, K., Rubin, S. G., and Khosla, P. K., "Laminar Natural Convection Along Vertical Square Ducts," Numerical Heat Transfer, Vol. 5, No. 1, 1982, pp. 59-79.

${ }^{5}$ Elenbaas, W., "Heat Dissipation of Parallel Plates by Free Convection," Physica, Vol. 9, No. 1, 1942, pp. 1-28.

${ }^{6}$ Miyatake, O., and Fujii, T., "Natural Convection Heat Transfer Between Vertical Parallel Plates at Unequal Uniform Temperatures," Heat TransferJapanese Research, Vol. 2, No. 4, 1973, pp. 79-88.

${ }^{7}$ Ofi, O., and Hetherington, H. J., "Application of the Finite Element Method to Natural Convection Heat Transfer from the Open Vertical Channel," International Journal of Heat and Mass Transfer, Vol. 20, No. 11, 1977, pp. $1195-1204$.

${ }^{8}$ Aihara, T., "Air Cooling Techniques by Natural Convection," Cooling Techniques for Computers, edited by W. Aung, Hemisphere, New York, 1991, pp. 1-45.

${ }^{9}$ Raithby, G. D., and Hollands, K. G. T., "Natural Convection," Handbook of Heat Transfer Fundamentals, 2nd ed., edited by W. M. Rohsenow, J. P. Harnett, and E. N. Ganic, McGraw-Hill, New York, 1985, Chap. 6, pp. 6-33-6-37.

${ }^{10}$ Churchill, S. W., and Usagi, R., "A General Expression for the Correlation of Rates of Transfer and Other Phenomenon," AIChE Journal, Vol. 18, No. 6, 1972, pp. 1121-1128.

${ }^{11}$ Shah, R. K., and London, A. L., Laminar Flow Forced Convection in Ducts, Academic Press, New York, 1978, pp. 200, 248, 263.

${ }^{12}$ Muzychka, Y. S., "Analytical and Experimental Study of Fluid Friction and Heat Transfer in Low Reynolds Number Flow Heat Exchangers," Ph.D. Dissertation, Dept. of Mechanical Engineering, Univ. of Waterloo, Ontario, Canada, Oct. 1999.

${ }^{13}$ Abramowitz, M., and Stegun, A., Handbook of Mathematical Functions, Dover, New York, 1970, pp. 587-626.

${ }^{14}$ Bar-Cohen, A., and Rohsenow, W. M., "Thermally Optimum Spacing of Vertical Natural Convection Cooled, Parallel Plates," Journal of Heat Transfer, Vol. 106, No. 1, 1984, pp. 116-123. 